\title{
Comparative Study on Method of Threshold Estimation: Distortion Product Threshold Test versus Pure Tone Threshold Test
}

\author{
Gunjan Mehta ${ }^{1}$ Anuj Kumar Neupane ${ }^{2}$ Abhishek Mistri ${ }^{1} \quad$ Hiral Joshi ${ }^{1}$ Nirmit Shah ${ }^{1}$ \\ ${ }^{1}$ Department of Audiology and Speech Therapy, C.U. Shah \\ Medical College and Hospital, Surendranagar, Gujarat, India \\ 2School of Audiology and Speech Language Pathology, \\ Bharati Vidyapeeth Deemed University, Pune, India \\ Address for correspondence Gunjan Mehta, MASLP, \\ Department of Audiology and Speech Therapy, C.U. Shah Medical \\ College and Hospital, Surendranagar 363001, Gujarat, India \\ (e-mail: gunjanmehta06@gmail.com).
}

\begin{abstract}
Keywords

- DPOAE

- pure tone

- thresholds

- input/output

functions

Introduction Distortion product otoacoustic emissions (DPOAEs) can be helpful in estimating the hearing thresholds by extrapolating the DPOAE input/output function with the help of scissor paradigm, and thus the following study aims at assessing its reliability by comparing DPOAE thresholds with those obtained by behavioral responses at pure tones on various frequencies.

Materials and Methods Fifty participants having normal hearing sensitivity were included in the study. Pure tone (PT) audiometry was carried out on all participants to determine PT thresholds in both ears. DPOAE threshold test was administered and thresholds at 1, 2, 4, and $8 \mathrm{kHz}$ were compared accordingly.

Results The result indicated significant difference between the two methods of threshold estimation where the mean difference was found to be 3 decibel $(\mathrm{dB})$ and $4 \mathrm{~dB}$ for left and right ears, respectively. Shapiro-Wilk test revealed normal distribution $(p>0.05)$ of the data. Hence, parametric paired $t$-test was performed, which revealed significant difference between PT and distortion product thresholds.

Conclusion Study concludes as having good clinical applicability in assessing neonates and infants.
\end{abstract}

\section{Introduction}

The clinical applications of otoacoustic emissions (OAEs) are well known in estimating hearing sensitivity., ${ }^{1,2}$ OAEs are of different subtypes ${ }^{3}$ out of which distortion product otoacoustic emissions (DPOAEs) are generated by cubic distortion $\left(2 \mathrm{f}_{1}-\mathrm{f}_{2}\right)$ of outer hair cells (OHCs) that leads to nonlinearity in selective frequency compression. ${ }^{4-6}$ When recorded at stimulus levels that are near to hearing levels, the sensitivity of cochlear amplifier (CA) can be reflected at its best. Quantitative and frequency-specific data regarding the range and operating features of the CA such as sensitivity,

DOI https://doi.org/ $10.1055 / \mathrm{s}-0041-1724218$ ISSN 2581-9607. compression, and frequency selectivity of the OHCs of cochlea can be obtained from DPOAE. ${ }^{7}$ DPOAE can be interpreted in two ways, which include distortion product (DP)-Gram and DPOAE input/output $(\mathrm{I} / 0)$ functions.

DPOAE I/O functions provide information on estimating behavioral threshold and so the rate of growth of cochlear responses. ${ }^{8-10}$ One can predict DPOAE's threshold, that is, decibel in hearing level (dBHL), by extrapolating DPOAE $\mathrm{I} / \mathrm{O}$ functions. This feature allows for the assessment of the cochlear sensitivity and compression. DPOAEs are acquired with frequency ratio of $\mathrm{f} 2 / \mathrm{f} 1$ at 1.2 and setting of two primary tone levels, L1 and L2. ${ }^{11}$ Along with the reduction in

\section{(C)2021. Indian Society of Otology.}

This is an open access article published by Thieme under the terms of the Creative Commons Attribution-NonDerivative-NonCommercial-License, permitting copying and reproduction so long as the original work is given appropriate credit. Contents may not be used for commercial purposes, or adapted, remixed, transformed or built upon. (https://creativecommons.org/licenses/by-nc-nd/4.0/).

Thieme Medical and Scientific Publishers Pvt. Ltd. A-12, 2nd Floor, Sector 2, Noida-201301 UP, India 
stimulus level, the variance in the primary tone level needs to be increased to reserve the peak intersection of primary tone traveling waves at a constant frequency ratio. This results in the decrease in L1 being lower than the decrease in L2, which is termed as scissor paradigm. ${ }^{11}$

Numerous authors have investigated predicting or estimating pure tone (PT) threshold by extrapolating DPOAEs. Study by Boege and Janssen ${ }^{9}$ estimated PT thresholds by extrapolating DPOAEs via scissor paradigm and therefore, it was compared with behavioral PT thresholds. It was found to have a mean difference of $2.2 \mathrm{~dB}$ between the extrapolated thresholds and behavioral PT thresholds. Furthermore, DPOAE thresholds across frequencies were extrapolated and were compared with behavioral PT thresholds. Significant correlation was found across all frequencies between DPOAE thresholds and behavioral PT thresholds. ${ }^{10}$ Study by Schmuziger et al ${ }^{12}$ compared PT thresholds with automated DPOAE thresholds, where nominal mean difference between PT thresholds and automated DPOAE thresholds was reported. Similarly, the pilot study done to administer DPOAE thresholds via Cochlea Scan device in normal hearing and individuals with hearing impairment revealed a stronger relationship between DPOAE thresholds and behavioral PT thresholds across both groups. ${ }^{13}$

Data from various studies have suggested minor differences in DPOAE thresholds as compared to behavioral PT thresholds signifying the applicability of DPOAE thresholds in estimating hearing thresholds in neonates and infants, where traditional behavioral responses are not reliable. ${ }^{5,14}$ DPOAE thresholds have been observed as efficient clinical tools in estimating hearing threshold in mild to moderate hearing losses as compared to auditory-evoked potential measures, especially in infants. ${ }^{13}$ Similar to Cochlea Scan device mentioned in various studies, PATH Medical Device has come up with latest DPOAE measures known as DP threshold based on scissor paradigm.

However, there is dearth of studies on comparison of DP threshold measure with PT thresholds. Therefore, the present study aimed at evaluating behavioral PT threshold estimation with the DP threshold estimation in normal hearing adults.

\section{Materials and Methods}

\section{Participants}

Simple random sampling was utilized for the study. A total of 50 individuals ( 100 ears) between the age range of 18 and 35 years (mean age: 22.08 ) with no history or present complaints of hearing loss or any related problems such as neurological or physical incompetence were selected for the study. Individuals with any history of ototoxic medication or overexposure to high noise levels were excluded from the study. All the participants were informed initially regarding the study and therefore, written consent was obtained. Bioethical clearance was achieved for the study as per the guidelines of the ethics committee of the institution.

\section{Procedure}

All tests were performed in an acoustic room setup with maximum permissible noise levels as specified by ANSI-S3.1
(1991). ${ }^{15}$ The subsequent procedures were followed during the study.

Otoscopic Examination: It was performed using Heine 3000 mini otoscope, and individuals with intact tympanic membrane and absence of impact ear wax were further included in the study.

Immittance Audiometry: Immittance audiometry was performed with Path Sentiero immittance device with EP-TY 9301769 transducer to rule out conductive component if any. Subjects with "A" type tympanogram were only included in the study.

Pure-Tone Audiometry (PTA): PTA was performed with GSI 61 Clinical Audiometer. Pure tones were presented using TDH-39 supra-aural headphones on octave frequencies of 250, 500, 1000, 2000, 4000, and $8000 \mathrm{~Hz}$ applying Hughson-Westlake method. ${ }^{15}$ PT average of each ear was derived from four frequencies: 500, 1000, 2000, and $4000 \mathrm{~Hz}$. Subjects having PT average of less than $15 \mathrm{~dB}$ were further administered with DPOAE.

DPOAE: Sentiero Advanced Device from Path Medical Solutions (IEC 60645-6:2009) was utilized in the study along with EP-DP Probe-SN4301780 transducer. Individuals with DPOAEs present at three consecutive frequencies were further selected for DP threshold test measures.

DP Threshold Test: DP threshold test module, developed and patented by Path Medical Solutions, directly measures the DPOAE threshold in dBHL. It is based on the "scissor" paradigm (i.e., $2 \mathrm{f}_{1}-\mathrm{f}_{2}$ ), which refers to optimized paradigms to yield robust detection of DPOAE responses and is used in $\mathrm{I} / \mathrm{O}$ function and DPOAE threshold estimation. The DPOAE threshold test has a measurement window of $50 \mathrm{dBHL}$ and is measured on $1,1.5,2,3,4,5,6$, and $8 \mathrm{kHz}$. In the present study, comparative analysis was done between PT thresholds and DP thresholds at 1, 2, 4, and $8 \mathrm{kHz}$ frequencies. DP thresholds across frequencies are obtained by plotting DPOAE I/O functions in DPOAE pressure, that is, $\mathrm{P}_{\mathrm{dp}}$. The decibel in sound pressure level at which the $\mathrm{P}_{\mathrm{dp}}$ is obtained as 0 Pascal (pa) is considered as the DP threshold. This is depicted in - Fig. 1.

\section{Results}

The present study aimed at comparing DP threshold across frequencies with PT thresholds. Descriptive statistics was done with IBM SPSS version 24 software, to calculate the mean and standard deviation (SD) of PT and DP thresholds across frequencies of all the participants as given in - Table $\mathbf{1}$.

In - Table 1 are depicted the mean and SD of PT thresholds and DP thresholds across different frequencies along with their mean differences. The mean threshold differences between PT thresholds and DP thresholds were found to be 2.24 ( $\mathrm{SD}=4.4), 2.8$ (SD = 5.3), 7.18 ( $\mathrm{SD}=6.68$ ), and 5.66 ( $\mathrm{SD}=7.98$ ) at $1 \mathrm{kHz}, 2 \mathrm{kHz}, 4 \mathrm{kHz}$, and $8 \mathrm{kHz}$, respectively, for right ear. Similarly, it was found to be 2.1 (SD = 4.7), 1.82 ( $\mathrm{SD}=4.6), 5.78(\mathrm{SD}=5.98)$, and $4.48(\mathrm{SD}=6.55)$ at $1 \mathrm{kHz}, 2$ $\mathrm{kHz}, 4 \mathrm{kHz}$, and $8 \mathrm{kHz}$, respectively, for the left ear. The DP thresholds and PT thresholds recorded for both ears in one of the participants are given in - Fig. 2 . 
$f_{2}=1 \mathrm{kHz}$

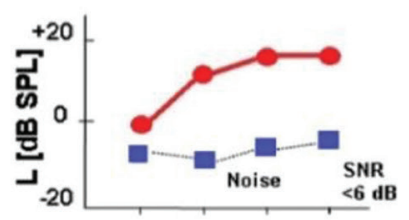

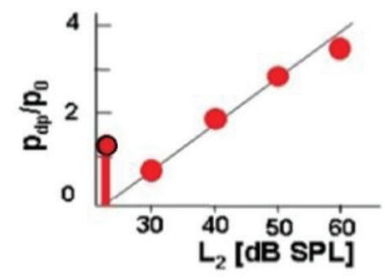

$f_{2}=3 \mathbf{k H z}$

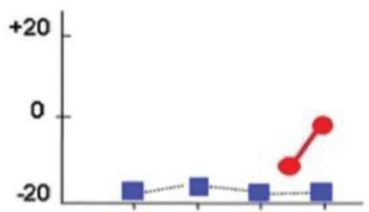

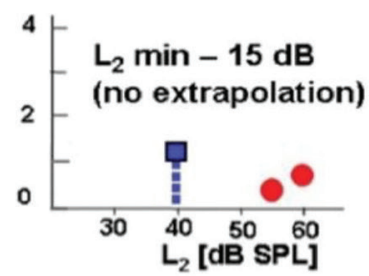

$$
f_{2}=2 \mathrm{kHz}
$$
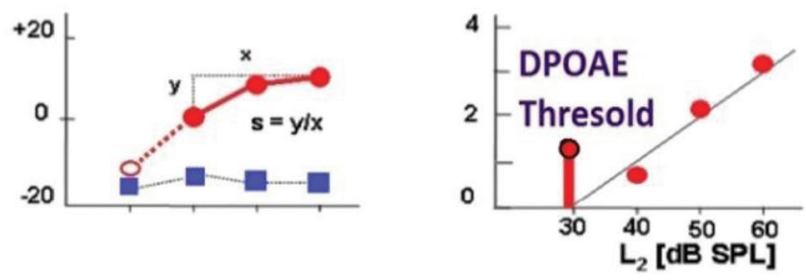

$f_{2}=6 \mathrm{kHz}$
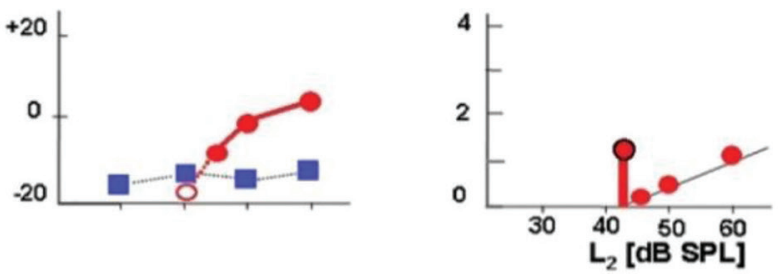

Fig. 1 Measures of extrapolation of distortion product otoacoustic emissions (DPOAE) and determination of DP threshold (retrieved from the user manual of the Path Medical Solutions). dB SPL, decibel in sound pressure level.

Table 1 Representation of the mean and standard deviation values for pure tone thresholds and distortion product thresholds at $1,000,2,000,4,000$, and $8,000 \mathrm{~Hz}$ in $\mathrm{dBHL}$

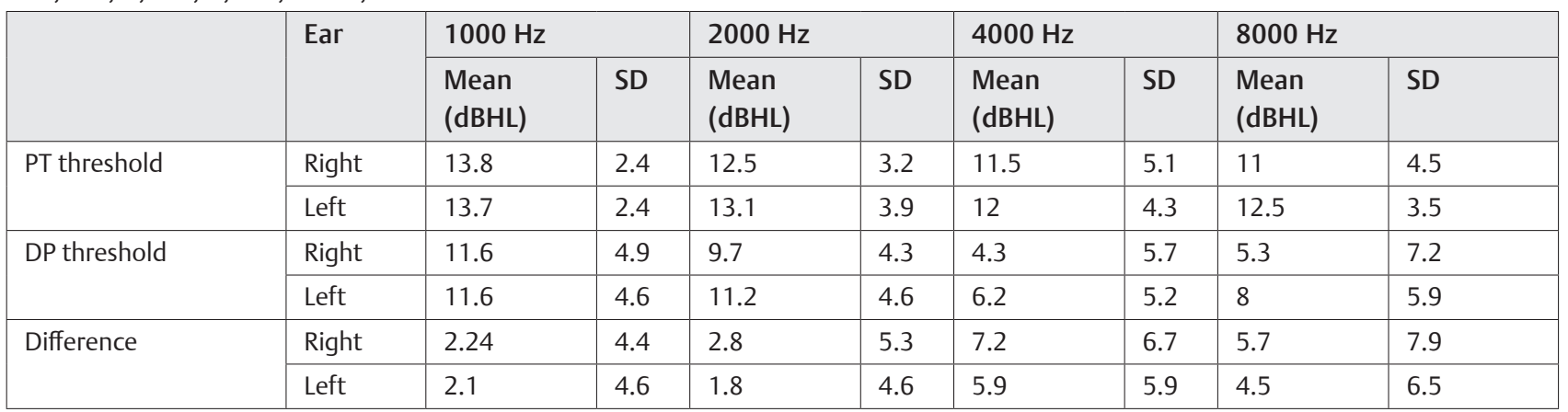

Abbreviations: $\mathrm{dBHL}$, decibel in hearing level; DP, distortion product; $\mathrm{Hz}$, Hertz; PT, pure tone; SD, standard deviation.

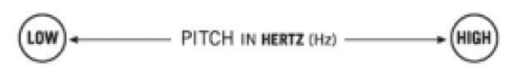

SOFT
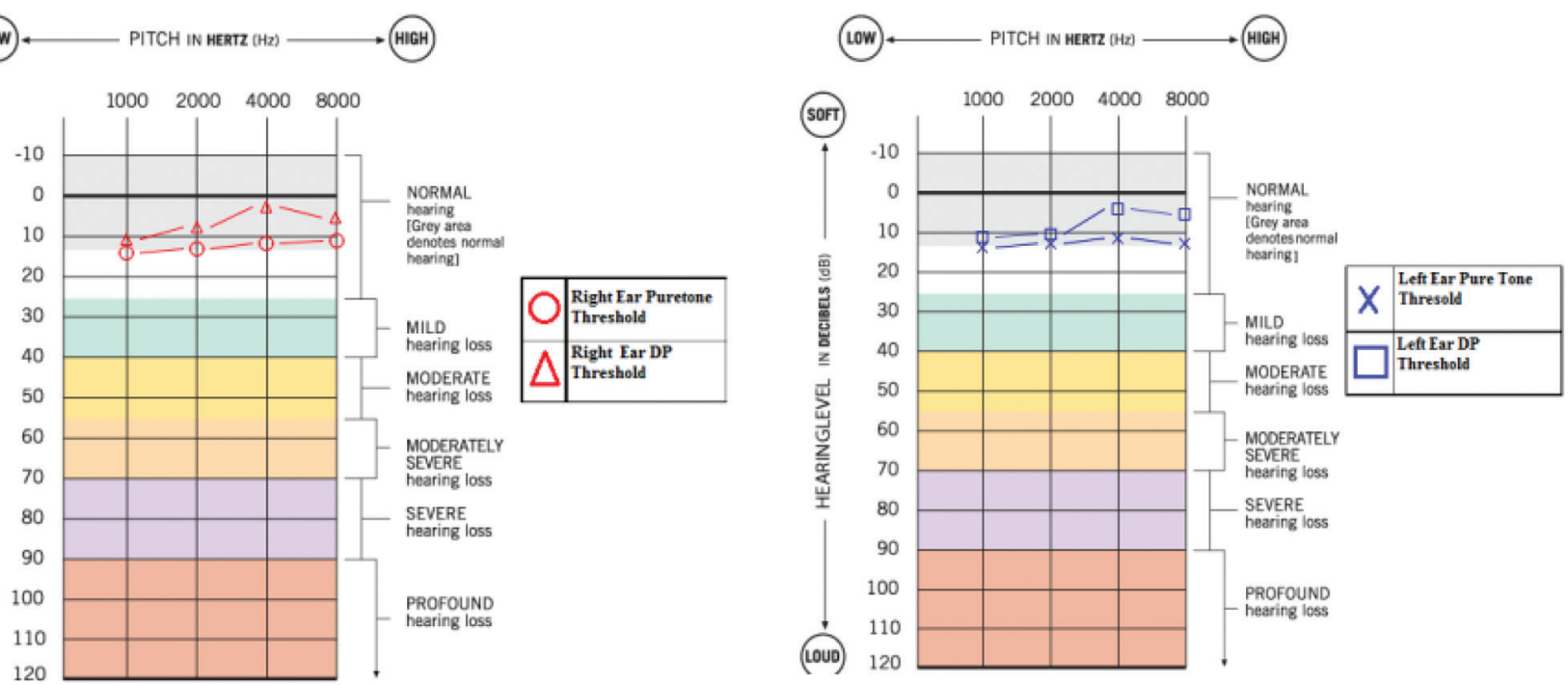

Fig 2 Comparative representation of distortion product (DP) thresholds and pure tone (PT) thresholds across frequencies in an individual with normal hearing sensitivity. 
Shapiro-Wilk test revealed normal distribution $(p>0.05)$ of the data. Hence, parametric paired $t$-test was performed, which revealed significant difference between PT and DP thresholds across $1 \mathrm{kHz}$ for left ear as $t(49)=3.17, p<0.05$, and right ear as $t(49)=3.6, p<0.05 ; 2 \mathrm{kHz}$ for left ear as $t(49)=$ $2.77, p<0.05$, and right ear as $t(49)=3.72, p<0.05 ; 4 \mathrm{kHz}$ for left ear as $t(49)=6.83, p<0.05$, and right ear as $t(49)=7.59$, $p<0.05$; and $8 \mathrm{kHz}$ for left ear as $t(49)=4.83, p<0.05$, and right ear as $t(49)=5.01, p<0.05$.

\section{Discussion}

The objective of the present study was to compare behavioral PT threshold estimation with the DP threshold estimation and check for variance, if any, between the two across different frequencies. To elaborate, the topic of interest was to check the dependability of DP threshold test in comparison to traditional behavioral methods for estimating PT thresholds. As it was the preliminary study, adult population was selected to ensure the consistency and accuracy of behavioral thresholds. The results indicated significant differences between DP thresholds and PT thresholds across frequencies.

In the present study, DP thresholds were found to have lower threshold values in dBHL than PT thresholds across test frequencies at 1, 2, 4, and $8 \mathrm{kHz}$. There was found to be significant difference between the two thresholds across frequencies. Overall, the mean difference between the thresholds was found to be $3 \mathrm{dBHL}$ for left ear and $4 \mathrm{dBHL}$ for right ear. The findings in the present study are concurrent with earlier studies. ${ }^{9}$ It was found that there was a mean difference of $2.2 \mathrm{~dB}$ for all data between estimated DPOAE thresholds and PT thresholds. In addition, Schmuziger et al ${ }^{12}$ reported an overall difference of $2 \mathrm{dBHL}$ ( $\mathrm{SD}=10$ ) between PTA and automated DPOAE threshold. Hence, automated DPOAE threshold was considered as an excellent test for clinical routine.

Present study also reported the mean differences between two thresholds to be more at higher frequencies as compared to mid frequencies. These results are equivocal with earlier studies where the mean differences between DPOAE thresholds and PT thresholds were found to be higher for mid-frequency thresholds as compared to lower and higher frequencies. ${ }^{10}$ Hatzopolous et $\mathrm{al}^{13}$ reported the mean variation in thresholds to be less than $8 \mathrm{dBHL}$ at 2,3 , and $4 \mathrm{kHz}$ in normal hearing individuals. Mean difference was found to be $9 \mathrm{dBHL}$ at $1.5 \mathrm{kHz}$, while behavioral threshold was overvalued by $2 \mathrm{dBHL}$ at $6.0 \mathrm{kHz} .{ }^{13,16}$ Comparatively larger variation in the high frequencies can be due to the presence of more robust DPOAE responses at these regions. ${ }^{7,10}$

\section{Conclusion}

The result from the present study provides normative and comparative data on DP thresholds for clinical usage and research. The study concludes as having significant difference between DP thresholds and PT thresholds, that is, $3 \mathrm{dBHL}$ in left ear and $4 \mathrm{dBHL}$ in right ear, which indicates applicability of the test used in clinical and research setup for assessment and verification of various otological conditions. This study finding develops the test applicability in assessing patients who are difficult to assess in the traditional test, especially infants and neonates. The limitations of the study could be its inability to assess lower frequencies for DP thresholds and hence could not be compared with PT thresholds. As it was the preliminary study, only young healthy populations were considered for the study. Therefore, the clinical applicability of the test was not carried out on individuals with hearing loss. These limitations can be considered as the future scope of the study.

\section{Author Contributions}

G.M.: Study design, data collection, data analysis, preparation of manuscript, and literature analysis. A.K.N.: Study design, data analysis, data interpretation, preparation of manuscript, and literature analysis. A.M.: Study design, data collection, preparation of manuscript, and literature analysis. H.J.: Study design, data collection, preparation of manuscript, and literature analysis. N.S.: Study design, data collection, preparation of manuscript, and literature analysis.

\section{Funding \\ None.}

\section{Conflict of Interest}

None declared.

\section{References}

1 Kemp DT. Otoacoustic emissions, their origin in cochlear function, and use. Br Med Bull 2002;63(1):223-241

2 Brownell WE. Outer hair cell electromotility and otoacoustic emissions. Ear Hear 1990;11(2):82-92

3 Gelfand SA, Wilson C. Book review: essentials of audiology. J Audiol Med 2001;10(3):216

4 Brownell WE, Bader CR, Bertrand D. de Ribaupierre Y. Evoked mechanical responses of isolated cochlear outer hair cells. Science 1985;227(4683):194-196

5 Janssen T, Klein A, Gehr DD. [Automated hearing threshold estimation in newborns using extrapolated DPOAE input/output functions]. HNO 2003;51(12):971-980

6 Kemp DT, Bray P, Alexander L, Brown AM. Acoustic emission cochleography-practical aspects. Scand Audiol Suppl 1986;25:71-95

7 Janssen T, Niedermeyer HP, Arnold W. Diagnostics of the cochlear amplifier by means of distortion product otoacoustic emissions. ORL J Otorhinolaryngol Relat Spec 2006;68(6):334-339

8 Martin GK, Probst R, Lonsbury-Martin BL. Otoacoustic emissions in human ears: normative findings. Ear Hear 1990;11(2):106-120

9 Boege P, Janssen T. Pure-tone threshold estimation from extrapolated distortion product otoacoustic emission I/Ofunctions in normal and cochlear hearing loss ears. J Acoust Soc Am 2002;111(4):1810-1818

10 Gorga MP, Neely ST, Dorn PA, Hoover BM. Further efforts to predict pure-tone thresholds from distortion product otoacoustic emission input/output functions. J Acoust Soc Am 2003;113(6):3275-3284

11 Kummer P, Janssen T, Hulin P, Arnold W. Optimal L(1)-L(2) primary tone level separation remains independent of test frequency in humans. Hear Res 2000;146(1-2):47-56 
12 Schmuziger N, Patscheke J, Probst R. Automated pure-tone threshold estimations from extrapolated distortion product otoacoustic emission (DPOAE) input/output functions. J Acoust Soc Am 2006;119(4):1937-1939

13 Hatzopoulos S, Ciorba A, Petruccelli J, et al. Estimation of pure-tone thresholds in adults using extrapolated distortion product otoacoustic emission input/output-functions and auditory steady state responses. Int J Audiol 2009;48(9):625-631
14 Klein A. Automated assessment of hearing threshold in neonates by means of extrapolated DPOAE I/O-functions (Doctoral dissertation, Technische Universität München).

15 Frank T. ANSI update: specification of audiometers. Am J Audiol 1997;6(3):29-32

16 Hatzopoulos S, Kochanek K, Sliwa L, Skarzynski H. A pilot study on assessing hearing threshold using the Cochlea-Scan. Med Sci Monit 2008;14(4):MT7-MT11 